\title{
Suplemento de selenio y zinc en la cubrición y preparto en ovejas Merino Australiano
}

\author{
Irabuena, ○@ ; Sterla, S. and Fernández Abella, D.
}

Polo de Producción y Reproducción en Rumiantes. CENUR Litoral Norte Salto. Universidad de la República. Uruguay.

\section{PaLABRAS CLAVE}

Oligoelementos.

Oveja.

Producción de cordero.

Reproducción.

\begin{abstract}
RESUMEN
Se estudió el efecto de la administración de un suplemento con Selenio y/o Zinc, sobre la fertilidad, prolificidad y producción de carne en ovejas Merinas Australianas pastoreando campo natural de basalto, en la Región Norte del Uruguay. Se diseñaron cuatro lotes: 1.Selenio (Se) inyectable, 2.- Zinc $(\mathrm{Zn})$ bolo intraruminal de óxido de zinc, 3.- Selenio-Zinc (SeZn), y grupo Control (C). Veintiún días precubrición se suplementó y, 21 días preparto se formaron subgrupos, alimentando a un subgrupo con $5 \mathrm{mg}$ de selenito de sodio por animal. Los grupos suplementados precubrición mostraron diferencia significativa en la fertilidad sobre el grupo C. Los lotes 1.- y 3.- presentaron el mayor porcentaje de partos respecto a los lotes 2.- y $C(p<0,05)$. El peso promedio de los corderos al nacimiento y destete no mostró diferencia entre los grupos. Al destete el sub-grupo suplementado preparto del tratamiento $Z n$, presentó mayor producción de carne total $(p<0,05)$. Los valores de Se y Zn en sangre previo a la administración del suplemento fueron carenciales, luego de la misma alcanzaron valores normales manteniéndose por 45 días para Se y 70 para Zn. En conclusión, la administración con Se y/o Zn en ovejas Merinas, presentó un efecto positivo sobre la fertilidad, el porcentaje de corderos destetados y la producción de carne
\end{abstract}

\section{Supplement of selenium and zinc in the covering and prepartum in Australian Merino sheep}

\section{SUMMARY}

The effect related to the administration of a supplement with Selenium and / or Zinc,on fertility, prolificacy and meat production in Australian Merino Ewes grazing natural basalt field, in the Northern Region of Uruguay was studied. Four lots of sheep were designated for each trail test: 1.- Injectable selenium (Se), 2.- Zinc (Zn) intraruminal bolus of zinc oxide, 3.- Selenium-Zinc (SeZn), and Controlled group (C). Twentyone days precoverage was supplemented and, 21 days prepartum, subgroups wereformed, feeding a subgroup with $5 \mathrm{mg}$ of sodium selenite per animal. The precoverage supplemented groups showed a significant difference in fertility over group C. Lots 1.-and 3.- presented the highest percentage of deliveries compared to lots 2.- and $C(p<0.05)$. The average weight of the lambs at birth and weaning showed no difference between the groups. At weaning, the predelivery supplemented, subgroup with $\mathrm{Zn}$ treatment presented a higher total meat production $(p<0.05)$. The values of $\mathrm{Se}$ and $\mathrm{Zn}$ in blood prior to administration of the supplement were low, affer which they reached normal values, remaining for 45 days on Se and 70 days on Zn. In conclusion, the administration of Se and / or Zn in Merino Ewes had a positive effect on fertility, the percentage of weaned lambs and meat production.

INFORMATION

Cronología del artículo.

Recibido/Received: 31.07 .2020

Aceptado/Accepted: 23.06 .2021

On-line: 15.07.2021

Correspondencia a los autores/Contact e-mail:

oirabu@gmail.com

\section{INTRODUCCIÓN}

En el Uruguay un claro indicador de la deficiencia reproductiva ovina, es el porcentaje de productividad, el mismo se ha mantenido entre un $60-70 \%$ promedio por más de una década. La tasa de productividad depende de la fertilidad y prolificidad de las ovejas servidas, y de la supervivencia neonatal (Salgado, 2014). Existe información de países de cría ovina extensiva como Australia y Nueva Zelanda que muestran la importancia de los microelementos en la reproducción y producción ovina. Cuando se produce un aporte mineral inadecuado o en proporciones incorrectas la función reproductiva es la más afectada, lo que conlleva a su vez, una disminución de la productividad y de la rentabilidad de las ganaderías (Tedó y Casas, 2005). Gabryszuk y Klewiec (2002) reportaron problemas relacionados a la carencia de selenio (Se) en ovinos: reducción de la fertilidad, abortos, retención de placentas y debilitamiento de corderos recién nacidos. Por su parte el zinc $(\mathrm{Zn})$, regula muchos de los efectos relacionados a la acción de las hormonas reproductivas y metabólicas del organismo. Por esta razón las deficiencias en este mineral pueden provocar, reducción en la secreción de gonadotrofina hipofisaria, disminución en la secreción de andrógenos, producción de óvulos no 
viables o fallas en la ovulación y en la maduración de ovocitos, así como retardo en el inicio de la pubertad y anormalidades fetales (Forero, 2004). Juega un rol importante en todas las fases del proceso reproductivo (estro, gestación y lactación (Smith and Akinbamijo, 2000) y es necesario para la implantación del embrión (Robinson et al., 2006).

Los efectos significativos de la deficiencia de Se y Zn ocurren en casos marginales donde los signos clínicos pueden no ser expresados (McDowell y Arthington 2005).

Existen claras evidencias de que los animales presentan mayores necesidades de Se y Zn durante la etapa reproductiva (Lopez Alonso et al. 1997, Chan et al. 1998).

A pesar de la importancia de estos microelementos, en el Uruguay es escasa la información existente de los efectos del suplemento con Se y Zn en ovinos, por ese motivo el objetivo del trabajo fue cuantificar las variaciones en la fecundidad y producción de carne (kg de cordero destetado/oveja), con el suplemento mineral de selenio y / o zinc de ovejas Merino Australiano, pastoreando campo natural de basalto superficial.

\section{MATERIALES Y METODOS}

\section{LOCALIZACIÓN DEL ENSAYO}

El trabajo de campo se realizó en un establecimiento ganadero, típicamente ovejero de la región Norte del Uruguay, ubicado en el departamento de Salto, $31^{\circ} 33^{\prime}$ latitud sur, 57 $50^{\prime}$ W). Dedicado a la cría de ovinos Merino Fino, con suelos de Basalto superficial, de uso netamente pastoril (MGAP-PRENADER, 2015).

\section{ANIMALES}

Se seleccionaron 21 moruecos Merino Australiano con características similares al examen de aptitud reproductiva (Fernández Abella, 2015) 694 ovejas Merino Australiano, homogéneas en condición corporal, peso vivo y edad (dentición) (3,2 $\pm 0,1$ unidades de escala corporal, $38,6 \pm 0,3 \mathrm{Kg}$ de peso vivo y 4 a 6 dientes), con medias para dichos valores $p>0,05$, fueron identificadas mediante crotales numerados. Los grupos experimentales estuvieron expuestos a iguales medidas de manejo sanitario, permanecieron en los mismos potreros (cercados) y pastorearon sobre campo natural.

\section{TRATAMIENTOS}

El ensayo consistió en suplementar con Se y/o Zn ovejas Merino Australiano en dos momentos diferentes, un suplemento tres semanas previo al comienzo del servicio a 694 ovejas, y otro a sub grupos de ovejas suplementadas en pre-cubrición que estaban preñadas, administrando Se tres semanas pre parto (Tabla I).

La administración de Se fue realizada vía subcutánea, a una dosis de $5 \mathrm{mg}$ /animal de selenito de sodio (Selfos Plus®, Laboratorios Codenor) y Zn, colocando un bolo ruminal de $\mathrm{ZnO}$ de $72 \mathrm{~g}$ por oveja, que libera $28 \mathrm{mg} \mathrm{Zn} / \mathrm{kg}$ peso vivo por día (The Time Capsule, AgResearch, New Zealand).
Tabla I. Suplemento pre-cubrición y pre-parto según tratamiento (Pre-coverage and pre-partum supplement according to treatment).

\begin{tabular}{|c|c|c|}
\hline Grupo & Pre-cubrición & Pre-parto \\
\hline \multirow{2}{*}{$\mathrm{Se}$} & \multirow{2}{*}{$5 \mathrm{mg} \mathrm{Se}(\mathrm{n}=174)$} & Se $5 m g(n=54)$ \\
\hline & & Control $(n=101)$ * \\
\hline \multirow[b]{2}{*}{ Se-Zn } & \multirow[b]{2}{*}{$5 \mathrm{mg}$ Se y $72 \mathrm{~g} \mathrm{Zn}(\mathrm{n}=172)$} & Se $5 m g(n=49)$ \\
\hline & & Control $(n=107)$ * \\
\hline \multirow[b]{2}{*}{$\mathrm{Zn}$} & \multirow{2}{*}{$72 \mathrm{~g} \mathrm{Zn}(\mathrm{n}=173)$} & Se $5 m g(n=54)$ \\
\hline & & Control $(n=98)$ * \\
\hline \multirow{2}{*}{ Control } & \multirow{2}{*}{$\begin{array}{l}\text { Sin Se y Zn * } \\
(n=175)\end{array}$} & Se $5 m g(n=48)$ \\
\hline & & Control $(n=92) *$ \\
\hline
\end{tabular}

*Estos grupos recibieron como placebo suero fisiológico isotónico.

\section{ANÁLISIS SANGUIINEOS}

Se extrajo sangre de la vena yugular, a 40 ovejas de cada tratamiento, los días -21 (21/3); 0 (10/4) y 75 (23/6), considerándose día 0 al comienzo de los apareamientos, una parte de la misma se trató con anticoagulante (EDTA) para la determinación de Selenio, (Marti et al. 2007), y otra fue centrifugada a $2500 \mathrm{rpm}$ durante 10 minutos, del suero obtenido se determinó zinc.

El selenio se determinó mediante la dosificación de glutatión peroxidasa (GSH-Px), según Ceballos et al. (1999), utilizando reactivo comercial de Laboratorios Randox, UK. Zinc mediante la técnica de espectrofotometría de absorción atómica con horno de grafito, (Whitehouse et al. 1982).

\section{PARÁMETROS REPRODUCTIVOS}

Los apareamientos se realizaron por servicio directo (3\% de moruecos) seleccionados según lo descrito anteriormente por Fernández Abella (2015). La fertilidad y carga fetal se determinaron mediante ultrasonografía, ecógrafo Wed 9618, con sondas rectal y convexa de 5,0 y 7,5 Mhz a los días 45 y 75 del comienzo del servicio.

Entre los días 145 y 184 se controló la parición durante 24 horas al día. Al parto se registró la fecha, sexo y peso del cordero al nacer. Se identificaron los corderos mediante crótalos numerados, y se identificaron las madres mediante números pintados en el costillar.

\section{Peso al NACER Y CRECIMIENTO DE LOS CORDEROS.}

Todos los corderos fueron pesados al nacimiento con una balanza Walmur $( \pm 0,050 \mathrm{~kg})$, permitiéndole que amamantaran antes, (Coates y Penning, 2000). Al destete (tres meses de edad), se registró peso de los corderos, se estimó el peso ajustado según fecha de nacimiento, para poder establecer comparaciones entre tratamientos. Diseño y tratamiento estadístico

El diseño experimental utilizado fue un Diseño Completamente Aleatorio, 694 ovejas homogéneas (edad, CC, peso), manejo sanitario y alimenticio fue igual para todas durante todo el ensayo. 
Dada la homogeneidad del material experimental se asignaron los tratamientos aleatoriamente a cada oveja. Se utilizó la siguiente fórmula

$$
y_{\mathrm{ij}}=\mu+\tau+\varepsilon
$$

Donde:

$y=$ observación del tratamiento " $\mathrm{i}$ ” en la repetición "j"; $\mu=$ media general; $\tau=$ efecto relativo del tratamiento i-ésimo; $\varepsilon=$ error experimental.

Para la descripción de las variables según tratamiento se utilizó ANOVA comparando efectos relativos de los tratamientos, considerando un modelo mixto, donde los tratamientos fueron los efectos fijos y las ovejas efectos aleatorios.

La variable preñez y el número de corderos nacidos y destetados fueron analizados según un modelo generalizado, ajustándose una distribución binomial. Las medias fueron comparadas con el test de Tukey ( $\alpha=5 \%)$ siempre que fue necesario. Se utilizó el software SAS (Montgomery, 2001).

\section{RESULTADOS Y DISCUSIÓN}

\section{VALORES DE GSH-PX Y ZINC}

Los valores promedio de GSH-Px y Zinc en sangre de las ovejas, previo al suplemento, fueron carenciales (Tabla II). Langlands et al. 1981, Gissel-Nielsen et al. 1984, McDowell, 1996, describen que el contenido y disponibilidad de Se y Zn en el suelo determinará su concentración en el forraje y éste a su vez regula la concentración sanguínea y tisular de los elementos en el animal a pastoreo. Los valores hallados muestran desbalances metabólicos para ambos elementos, sin la presentación de signos clínicos relacionados con la deficiencia. Al día cero del cronograma el valor promedio de GSH-Px de los grupos Se y Se$\mathrm{Zn}$, lograron valores adecuados (231,14 y 186,66 UI/ $\mathrm{gHb}$ respectivamente), siendo $\mathrm{p}<0,05$ con los grupos no suplementados, (73,48 y 74,31 Zn y Control respectivamente). Al día 45 del cronograma el valor de GSH-Px se mantuvo en niveles superiores $(p<0,05)$ de los grupos que recibieron Se respecto a los que no recibieron, con valores en rango marginal para el grupo Se $(119,43 \mathrm{UI} / \mathrm{gHb})$ y marginal bajo para el grupo Se-Zn $(98,94 \mathrm{UI} / \mathrm{gHb})$ (Tabla II).

Según Levander (1986) y Knight y Sunde (1988) el aumento en la actividad sanguínea de GSH-Px ocurre en un lapso de 1 a 4 semanas, ya que el aumento de la concentración orgánica de Se no conduce a la síntesis inmediata de la enzima, sino que está asociada a su incorporación a los eritrocitos en el proceso de eritropoyesis. Este aumento nos indica que la dosis empleada fue adecuada para superar la deficiencia de Se. Acorde a la vida media de los eritrocitos, la actividad de GSHPx medida en sangre refleja el balance nutricional de Se de los últimos 2 meses, período en el cual las ovejas sólo recibieron una dosis de selenio y luego pastorearon campo natural. En ese período el selenio administrado fue metabolizado y al no mantenerse el aporte y tener una dieta carencial en dicho elemento, comenzó a descender el nivel sanguíneo, disminuyendo a valores por debajo de lo adecuado al cabo de dos meses. Según Lopez Alonso et al. (1997), Gabryszuk y Klewiec, (2002) en la etapa de concepción, gestación pre y posparto, la deficiencia de Se reviste la mayor importancia sanitaria-productiva asociado al estrés oxidativo en ovejas. Los ovinos se adaptan a cantidades mínimas de Se en el ecosistema, como se refleja en las bajas concentraciones séricas del elemento, sin manifestar signos clínicos de dicha deficiencia. Muffarrege (1999) considera para la Argentina, que la administración de Se parecería ser una técnica que tiene buenas perspectivas en la reproducción de rumiantes. Por otra parte, Barcellos et al. (2003) afirman que para Río Grande do Sul, raras veces la carencia del elemento provoca la enfermedad del músculo blanco, pero ocurren deficiencias subclínicas que frecuentemente afectan la eficiencia de producción, reproducción y salud de los animales. Al día 21 post administración del suplemento el valor medio de zinc en sangre para las ovejas de los grupos Se-Zn y $Z n$ fue de 1,81 y 1,85 $\mu \mathrm{g} / \mathrm{mL}$ respectivamente y significativamente diferente $(\mathrm{p}<0,05)$ respecto a los otros grupos. Al día 45 la media de los valores de zinc para los grupos $\mathrm{Zn}$ y Se-Zn, presentaron valores dentro del rango normal 1,22 y 1,02 $\mu \mathrm{g} / \mathrm{mL}(\mathrm{p}<0,05)$ respecto de los grupos no suplementados, 0,42 y 0,40 $\mu \mathrm{g} / \mathrm{mL}$ Se y $C$ respectivamente. Los bolos de liberación lenta permiten mantener niveles de zinc en el tracto digestivo durante 60 días aproximadamente. Menard y Cousin (1983), encontraron que la velocidad de absorción del zinc por parte del intestino delgado era el doble en ratas deficientes que en aquellas que eran suficientes, debido a un aumento en el número de receptores que pueden capturar el elemento y conservar durante un período de tiempo su capacidad de absorción aumentada.

Kincaidy Cronrath (1979), indican que al suplementar con zinc terneros deficientes, la capacidad

Tabla II. Medias de valores de GSH-Px y zinc en sangre en diferentes momentos según tratamiento (Mean values of GSH-Px and zinc in the blood at different times according to treatment).

\begin{tabular}{lcccccc}
\hline Tratamiento & \multicolumn{3}{c}{ GSH-Px Ul/gHb } & \multicolumn{3}{c}{ Zn ug/mL } \\
& Día -21 & Día 0 & Día 45 & Día -21 & Día 0 & Día 45 \\
Se & $71,58 \mathrm{a}$ & $231,14 \mathrm{a}$ & $119,43 \mathrm{a}$ & $0,33 \mathrm{a}$ & $0,41 \mathrm{~b}$ & $0,42 \mathrm{~b}$ \\
Se-Zn & $73,56 \mathrm{a}$ & $186,66 \mathrm{a}$ & $98,94 \mathrm{a}$ & $0,37 \mathrm{a}$ & $1,81 \mathrm{a}$ & $1,22 \mathrm{a}$ \\
Zn & $74,95 \mathrm{a}$ & $73,48 \mathrm{~b}$ & $73,31 \mathrm{~b}$ & $0,32 \mathrm{a}$ & $1,85 \mathrm{a}$ & $1,02 \mathrm{a}$ \\
Control & $74,34 \mathrm{a}$ & $73,31 \mathrm{~b}$ & $74,17 \mathrm{~b}$ & $0,37 \mathrm{a}$ & $0,41 \mathrm{~b}$ & $0,40 \mathrm{~b}$ \\
\hline
\end{tabular}

Letras diferentes $(p<0,05)$. Valores normales superior a $130 \mathrm{Ul} / \mathrm{gHb}$ para GSH-Px y 0,8-1,2 ug/mL para Zn. 
de unión del zinc al plasma se reducía a medida que transcurría el tiempo y los valores sanguíneos descendían. Por su parte Suttle (2010), postula que la homeostasis del Zn es controlada a nivel de la absorción intestinal y no se reconoce un exclusivo órgano de reserva, los tejidos no tienen la capacidad de mantener una zinquemia en niveles fisiológicos en el largo plazo, cuando la carencia en la ingesta es sostenida. Esto concuerda con lo encontrado en el experimento donde las ovejas previo al suplemento estaban en nivel carencial, luego logran valores adecuados y vuelven a descender dichos valores. Al día 45 los resultados ecográficos mostraron que las ovejas del grupo Se presentaron el mayor número de animales preñados $60,5 \%$, y el grupo control el menor 33,7\% (p<0,05\%); 54,4\% y 42,8\% para los grupos Se-Zn y Zn respectivamente (Tabla III).

Al finalizar la cubrición no se encontró diferencia significativa ( $p>0,05)$ entre los grupos suplementados, pero sí de éstos con el grupo control. Estos resultados concuerdan con lo observado por Wattiaux (1995), que describe que la acción antioxidante de la GSH-Px, se refleja en la ovulación, protegiendo al oocito del daño oxidativo generado por los procesos intrínsecos de la ruptura folicular y de la acción de enzimas proteolíticas presentes en el lumen del cuerno uterino. En casos de deficiencia de selenio se pueden observar incremento en el porcentaje de abortos y muerte embrionaria temprana, número de natimuertos, así como incremento en la presentación de ovarios quísticos, celos silentes o erráticos. En el mismo sentido, McDowell et al. (1997), trabajando con vacas que pastorearon pastizales con bajos niveles de selenio y otras suplementadas con selenio aumentó la tasa de concepción del 49 al 76\%, y encontraron diferencias significativas cuando compararon las pérdidas embrionarias a los 30 días de gestación entre las hembras gestante no tratadas y las suplementadas con selenio. Hemingway (2003), suplementando ovejas con selenio logro incrementos en la fertilidad, debido al mayor número de espermatozoides adheridos a la pelúcida, como consecuencia de un número mayor de las contracciones uterinas que favorecen el ascenso espermático.

El mayor porcentaje de nacimientos se observó en los grupos suplementados con Se y SeZn (93\%), control $(84 \%)$ y Zinc $(77 \%)$. El porcentaje de corderos destetados fue significativamente superior para el grupo selenio respecto al grupo control 76 vs $58 \%$ respectivamente (Tabla IV).

El promedio de los pesos de los corderos al nacimiento fue: Zn 4,52 Kg, Se-Zn 4,49 Kg, Se 4,44 Kg y C $4,37 \mathrm{Kg}$, entre grupo Zn y Control $(\mathrm{p}<0,05)$. Tabla V. Los pesos promedios de los corderos al nacimiento se encuentran dentro de lo definido como peso óptimo para la raza Merino $(4,200-4,800 \mathrm{Kg})$ por Fernández Abella (1995). Al destete no se observó diferencia en el promedio de peso de los corderos entre los grupos (Tabla V), esto concuerda con lo hallado por Ganzábal y Echevarría (2005). La determinación del peso al destete se corrigió de acuerdo a la edad de los corderos, esta variable no fue significativa al destete ya que hay factores que afectan al animal en las primeras semanas o meses de vida y que posteriormente a medida que el cordero crece se van reduciendo. $\mathrm{El}$ ambiente donde
Tabla III. Datos ecográficos según tratamientos días 45 y 75 (Ultrasound data according to treatments days 45 and 75$)$.

\begin{tabular}{lllll}
\hline Grupo & Se & Se-Zn & Zn & Control \\
Total de ovejas & 177 & 171 & 173 & 175 \\
Gestando día 45 & $60,5 \%$ a & $54,4 \%$ ab & $42,8 \%$ bc & $33,7 \%$ c \\
Gestando día 75 & $96,2 \%$ a & $94,2 \%$ ab & $94,2 \%$ ab & $85,6 \%$ c \\
\hline
\end{tabular}

Letras diferentes indican diferencias significativas $(p<0,05)$.

Tabla IV. Porcentaje de corderos nacidos y destetados según tratamiento (Percentage of lambs born and weeded according to treatment).

\begin{tabular}{lcccc}
\hline Variable & Se & Se-Zn & Zn & Control \\
$\%$ corderos nacidos & $93 \mathrm{a}$ & $93 \mathrm{a}$ & $77 \mathrm{c}$ & $84 \mathrm{~b}$ \\
$\%$ corderos destetados & $76 \mathrm{a}$ & $63 \mathrm{ab}$ & $62 \mathrm{ab}$ & $58 \mathrm{c}$ \\
\hline
\end{tabular}

Letras diferentes $p<0,05$.

Tabla V. Peso de los corderos al nacer, al destete y producción total de carne (Weight of lambs at birth, weeding and total meat production).

\begin{tabular}{|c|c|c|c|c|}
\hline Variable (Kg) & $\mathrm{Se}$ & Se-Zn & $\mathrm{Zn}$ & Control \\
\hline $\begin{array}{l}\text { Peso } \\
\text { nacimiento }\end{array}$ & $4,440 a b$ & $4,490 \mathrm{ab}$ & 4,520 a & $4,370 \mathrm{~b}$ \\
\hline $\begin{array}{l}\text { Peso } \\
\text { destete }\end{array}$ & $17,190 \mathrm{a}$ & 17,260 a & $17,550 \mathrm{a}$ & $17,180 \mathrm{a}$ \\
\hline $\begin{array}{l}\text { Producción } \\
\text { total carne }\end{array}$ & $17,190 \mathrm{~b}$ & $17,540 a b$ & $18,150 \mathrm{a}$ & $17,180 \mathrm{~b}$ \\
\hline
\end{tabular}

Letras diferentes $p<0,05$.

pastorearon las ovejas y se desarrollaron los corderos fue similar para todos.

La producción total de carne ( $\mathrm{kg}$ de cordero por oveja) fue mayor para el caso del grupo con suplemento de Zn (18,150 Kg), seguido de Se-Zn (17,540); Se $(17,190)$ y Control $(17,180)$, Zn respecto a los grupos Se y Control presenta $\mathrm{p}<0,05$ (Tabla V).

Durante la gestación tanto la madre como el feto son muy susceptibles a desequilibrio de micronutrientes de la dieta (Ghany-Hefnawy et al. 2007). Se sabe que el déficit de zinc durante la gestación en humanos, tiene una repercusión negativa en el sistema endócrino, condicionando entre otras manifestaciones clínicas, un fallo en el crecimiento (Milner, 1990), y que la administración de suplementos de zinc logra mejorar la tasa de crecimiento pos nacimientos en estos pacientes (Cheruvanky et al. 1982). Los estudios en animales en etapa pos natal deficientes en $\mathrm{Zn}$, han demostrado que uno de los mecanismos implicados en el retardo del crecimiento y bajo peso es la baja concentración de los niveles circulantes de IGF-I, que se incrementan cuando seaporta zinc (Cheruvanky et al. 1982). Según Ahola et al. (2004) los kilogramos de la progenie destetada por hembra expuesta a un suplemento con oligoelementos pueden verse afectada tanto por el suplemento mineral traza como por la fuente, esto podría explicar en parte los resultados obtenidos en este experimento. 
Al estudiar los lotes según el suplemento preparto con Se no se observó diferencia en el peso de los corderos al nacimiento, no se observó diferencias en el número de corderos nacidos muertos, así como la supervivencia neonatal.

Se produjo en promedio más kilos de carne por oveja para el caso de las ovejas del grupo zinc que recibieron selenio preparto respecto al resto de los tratamientos $(\mathrm{p}<0,05)$ (Tabla VI).

\section{CONCLUSIONES}

Bajo nuestras condiciones de trabajo podemos formular las siguientes conclusiones La concentración de selenio y zinc en sangre en las ovejas son carenciales. Con el protocolo utilizado, se logró valores sanguíneos adecuados de los elementos en estudio.El suplemento de selenio pre-cubrición mejoró los porcentajes de preñez y de corderos. Asimismo, el agregado de selenio en el grupo Zinc produjo más Kg de carne por oveja. Estos resultados obtenidos indican que se pueden mejorar la productividad y rentabilidad de la producción ovina, en la región basáltica del Uruguay.

\section{AGRADECIMIENTOS}

Proyecto financiado por Comisión Sectorial de Investigación Científica, Universidad de la Republica. Proyecto de Relacionamiento con el Sector Productivo. Modalidad 2.

\section{BIBLIOGRAFÍA}

Ahola, J, Baker, D, Burns, P, Mortimer, R, Enns, R, Whittier, J, Geary, T, \& Engle, T 2004, 'Effect of copper, zinc, and manganese supplementation and source on reproduction, mineral status, and performance in grazing beef cattle over a two-year period', Journal Animal Science, vol. 82, no 8, pp. 2375-83.

Barcellos, J, Wunsch, C, Prates, E, \& Ospina, H 2003, 'Suplementação mineral de bovinos de corte em ambientes subtropicais'. In: Barcellos, JOJ et al. (Ed). Suplementação Mineral de Bovinos em Regiões Subtropicais. Ed. UFRGS Porto Alegre, Brasil, pp. 19-51.

Ceballos, A, Wittwer, F, Contreras, P, \& Böhmwald, E 1999, 'Actividad de glutatión peroxidasa en bovinos lecheros a pastoreo correlacionada con la concentración sanguínea y plasmática de selenio', Pesquisa Agropecuária Brasileira, vol. 34, no 12, pp. 2331-2338.

Coates, D \& Penning, P 2000, 'Measuring animal performances'. In: ' $\uparrow$ Mannetje L. \& Jones R.M., eds. Field and laboratory methods for grassland and animal production research. Wallingford, UK: CABI Publishing, pp. 353- 402.

Chan, S, Gerson, B, \& Subramaniam, S 1998, 'The role of copper, molybdenum, selenium, and zinc in nutrition and health', Clinics in Laboratory Medicine, vol. 18, pp. 673-685.

Cheruvanky, T, Castro-Magana, M, Chen, S, Collipp, P, \& GgavamiMoibodi, Z 1982, 'Effect of growth hormone on hair, serum, and urine zinc in growth hormone-deficient children', American Journal of Clinical Nutrition, vol. 35, no 4, pp. 668-670

Fernández Abella, D 1995. 'Temas de reproducción ovina e inseminación artificial en bovinos y ovinos', Montevideo, Universidad de la República, 206 pp.

Fernández Abella, D 2015, 'Tecnologías reproductivas bovinas y ovinas', Montevideo. Uruguay. Edición Hemisferio Sur, 200 pp.

Forero, L 2004. 'Fallas reproductivas asociadas a deficiencias de microminerales, caso colombiano', http://www.produccionanimal. com.ar/suplementacion_mineral/12 2deficiencias_micorminerales_colombia.pdf 14/8/2018.

Gabryszuk, M \& Klewiec, J 2002, 'Effect of injecting 2- and 3-year-old ewes with selenium and selenium-vitamin $\mathrm{E}$ on reproduction and rearing of lambs', Small Ruminant Research, vol. 43, no. 2, pp. 127-132.

Ghany-Hefnawy, A, López-Arellano, R, Revilla-Vázquez, A, RamírezBribiesca, E, \& Tórtora-Pérez, J 2007, 'The relationship between fetal and maternal selenium concentrations in sheep and goats', Small Ruminant Research, vol. 73, pp. 174-180.

Ganzábal, A \& Echevarría M 2005, 'Análisis comparativo del comportamiento reproductivo y habilidad materna de ovejas cruza', Seminario de 390. Reproducción Ovina, INIA Uruguay, Serie de Difusión 401, abril - mayo 2005, pp. 33-42.

Gissel-Nielsen, G, Gupta, U, Lamand, M, \& Westermack, T 1984, 'Selenium in soils and plants, and its importance in livestock and human nutrition', Advances in Agronomy, vol. 37, pp. 397-460.

Hemingway, R 2003. 'The influences of dietary intakes and supplementation with selenium and vitamin $E$ on reproduction diseases and reproductive efficiency in cattle and sheep', Veterinary Research Communications, vol. 27 no. 2, pp 159-174.

Kincaid R, \& Cronrath J 1979, 'Effect of dietary zinc upon tissue zinc and percent unsaturated plasma zinc binding capacity', Journal Dairy Science, vol. 62, no. 4, pp. 572-576.

Knight, S, \& Sunde, R 1988, 'Effect of selenium repletion on glutathione peroxidase protein level in rat liver', Journal Nutrition, vol. 118 , no. 7, pp. 853-858.

Langlands, J, Bowles, J, Smith, A, \& Donald, G 1981, 'Selenium concentration in the blood of ruminants grazing northern New South Wales. II. Relationship with geological, pedological and other variables', Australian Journal of Agricultural Research, vol. 32, pp. 523-533.

Levander, O 1986, 'Selenium', In: Mertz, W. Trace elements in human and animal nutrition. 5th.ed., vol. 2. Academic Press, Inc. Orlando, USA, pp 209- 275

Lopez Alonso, M, Miranda, M, Hernandez, J, Castillo, C, \& Benedito, J 1997,

'Glutatión peroxidasa (GSH-Px) en las patologías asociadas a deficiencias de selenio en rumiantes', Archivo Médico Veterinario, vol. 29, no. 2, pp. 171-180.

Marti, E, Mara, L, Marti, J, Muiño-Blanco, T, \& Cebrián-Perez, J 2007, 'Seasonal variations in antioxidant enzyme activity in ram seminal plasma', Theriogenology, vol. 67, no. 9, pp. 1446-1454.

McDowell, L, \& Arthington, J 2005, 'Minerales para rumiantes en pastoreo en regiones tropicales', $2^{a}$ Edición Universidad de la Florida, Gainesville, IFAS, USA, pp: 6-47.

McDowell, L, Valle, G, Rojas, L, \& Velásquez-Pereira, J 1997, 'Importancia de la suplementación mineral completa en la reproducción de vacas', In: XXXIIII Reunión nacional de investigación pecuaria, XXIII Simposium de ganadería tropical: Interacción nutrición-reproducción en ganado bovino, Veracruz, México, pp. 31-47.

McDowell, L 1996, 'Feeding minerals to cattle on pasture', Animal Feed Science and Technology, vol. 60, pp. 247-271.

McDowell, L 2003, Minerals in Animal and Human Nutrition, Second Edition. Elsevier Science B. V., Amsterdam, The Netherlands, 644 pp. Menard, M, \& Cousins R 1983, 'Zinc transport by brush border membrane vesicles from rat intestine', Journal Nutrition, vol.113, no. 7, pp. 1434-1442.

Ministerio de Ganadería y Pesca, MGAP-PRENADER, Dirección General de Recursos Naturales, Uruguay, In: https://www.gub.uy/ministerioganaderia-agricultura-pesca/tramites-y-servicios/servicios/consultaconeat, 15/02/2018

Milner, J 1990, 'Trace minerals in the nutrition of children', Journal Pediatric,vol. 117, no. 2, pp. 145-155.

Montgomery D 2001, 'Design and Analysis of Experiments', Arizona State University, John Wiley \& Sons, Inc New York. Fifth Edition, 104 pp.

Mufarrege, D 1999, 'Los minerales en la alimentación de vacunos de carne en la Argentina', Trabajo de Divulgación Técnica Estación 
Experimental Agropecuaria INTAMercedes, Corrientes Argentina, In: www.produccion- animal.com.ar, 36 pp, 28/01/2018.

Robinson, J, Ashworth, C, Rooke, J, Mitchell, L, \& McEvoy, T 2006, Nutrition and fertility in ruminant livestock, Animal Feed Science and Technology, vol. 126, no. 3-4, pp. 259-276.

Salgado, C 2014, 'El Mercado de carne ovina en Lana noticias', Lana Noticias, vol. 166, pp. 21-23.

Smith, O, \& Akinbamijo, O 2000, Micronutrients and reproduction in farm animals, Animal Reproduction Science, vol. 60-6] , pp. 549-560.

Suttle, N.F 2010, The mineral nutrition of livestock, $4^{\text {th }}$ Edition. CABI International, Wallingford, UK, pp. 54-167.

Tedó, G \& Casas, J 2005, 'Importancia de los aportes de microminerales en la dieta de ganado ovino', TEGASA, Departamento Técnico Ru- miantes, http://www.tegasa.es/noticias/importancia-de-los-aportesde-microminerales-en-la-dieta-del-ganado-ovino.html 20/1 1/2018.

Wattiaux, M 1995, Reproducción y nutrición, Instituto Babcock para la investigación y desarrollo Internacional de la Industria Lechera, Universidad de Wisconsin, Madison, Wisconsin.

Whitehouse, R, Prasad, A, Rabbani, P \& Cossack, Z 1982, 'Zinc in plasma, neutrophils, lymphocytes, and erythrocytes as determined by flameless atomic absorption spectrophotometry', Clinical Chemestry, vol. 28 , no. 3 , pp. $475-480.1$ 